\title{
Evolução de florestas amazônicas inundadas, de igapó - um exemplo do rio Tarumà - Mirim(*)
}

\begin{abstract}
Resumo
Este trabalho tenta compreender o comportamento dos artrópodos do solo da fioresta de inundação do rio Tarumã-Mirim, perto de Manaus, Amazonas, em relação à evolução sedimentológica durante o período quaternário.
\end{abstract}

\section{INTRODUÇÃo}

As florestas que sofrem inundação no rio Tarumã-Mirim, perto de Manaus, Amazonas, apenas recentemente têm sido objeto de vários estudos botânicos, limnológicos e ecoetológicos (Adis, 1977; Adis \& Rodrigues, s/d; Beck. 1969, 1971, 1972 e 1976; Irmler, 1975, 1976, 1978 e s/d; Montgomery et al., s/d; Schaller, 1969 e 1973). Estudos terrestres forçosamente intervêm com as adaptações de artrópodos do biótopo "florestas inundadas".

Até agora, os resultados mostraram que os artrópodos do solo migram para as copas das árvores antes da inundação da floresta (Adis, 1977; Irmler, s/d) ou passam o período de inundação debaixo da água, no estágio de ovos (Beck, 1969).

Foi feita uma tentativa para compreender esse comportamento em relação à evolução sedimentológica do rio Tarumã-Mirim, durante o período quaternário.

\section{ÁREA DE ESTUdo E MÉTODOS DE INVESTIGAÇõES}

As amostras foram coletadas na área de floresta do rio Tarumã-Mirim, um tributário do rio Negro (profundidade média da água $=$

\author{
Georg Irion ( ${ }^{\#)}$ \\ Joachim Adis ("*)
}

$21 \mathrm{~m}$ ), aproximadamente $20 \mathrm{~km}$ acima de Manaus (Fig. 1 e 2). Em 4 pontos, foi medida a profundidade da água, obtendo-se uma profundidade máxima (durante a cheia) entre 1,5 a 5,5 metros. No momento da coleta de amostras, o nivel da água era de cerca de 3 metros mais baixo do que na época em que foram realizadas as medidas de profundidade da água. Como os sedimentos eram muito semelhantes, foi investigada apenas uma sonda. gem de $10 \mathrm{~m}$ de profundidade $(=$ ponto 3 vid. it mler, 1975, e ponto F vide Adis \& Rodrigues, $\mathrm{s} / \mathrm{d})$. As amostras foram submetidas à separação granulométrica. $A$ fração $<2 \mu \mathrm{m}$ foi analisada através de raio-x.

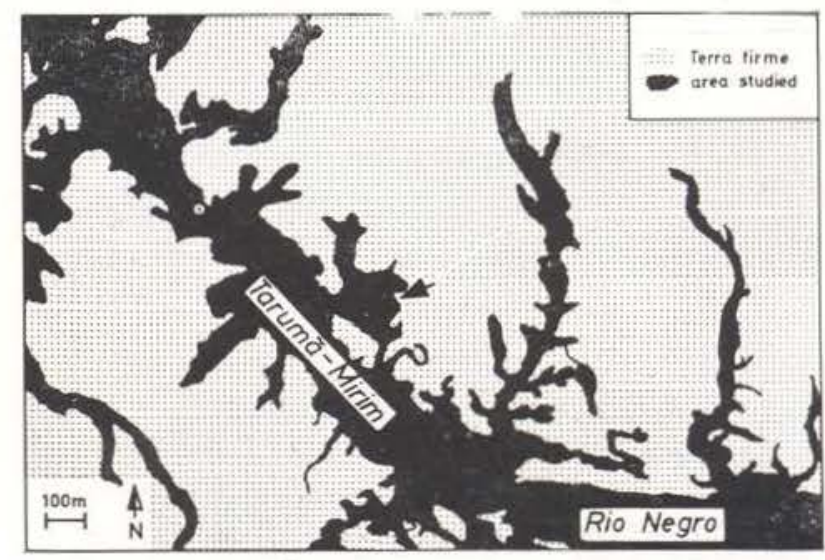

Fig. 1 - Localização da área estudada.

\section{REsultados}

A Fig. 3 mostra a distribuição granulométrica de 4 unidades selecionadas da sondagem. A mais profunda das três amostras contém

(*) - Por cooperaçâo enue Senckenberg-Institut Wilhemshaven, Alemanha, Instituto Nacional de Pesquisas da - Amazônia, Manaus, Brasil, e Max-Planck-Institut für Limnologie, Plön, Alemanha. Este estudo foi subsidi do pelo Deutsche Forschumgsgemeischaft (Aktenzeichen Ir 6/2). Agradecimento ao INPA (Projeto OEA) e ao Studienstiftung des Doutschen Volks.

(") - Max-Planck-Institut für Limnologie, Plön, Alemanha 


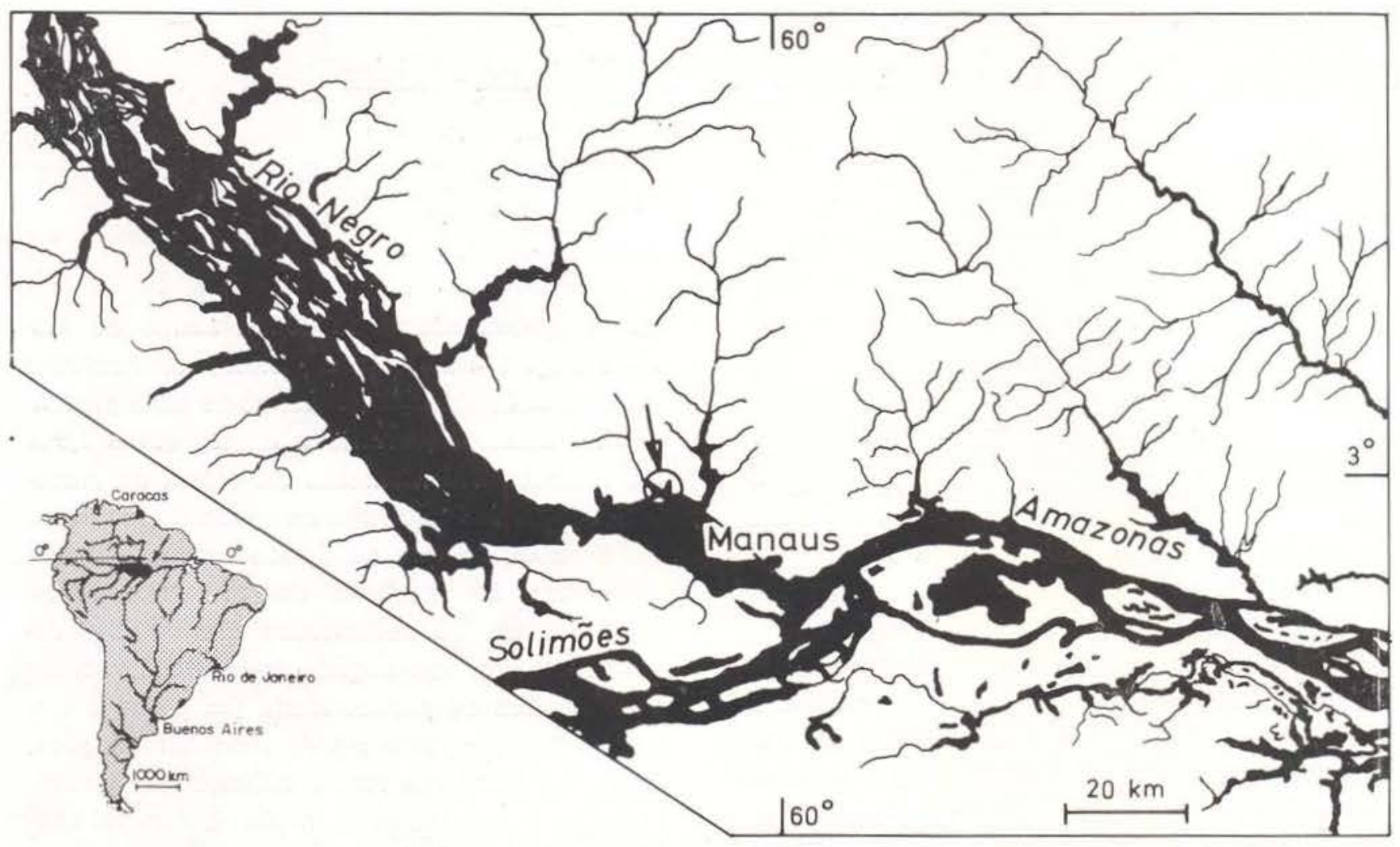

Fig. 2 - Localização geográfica da Fig: 1.

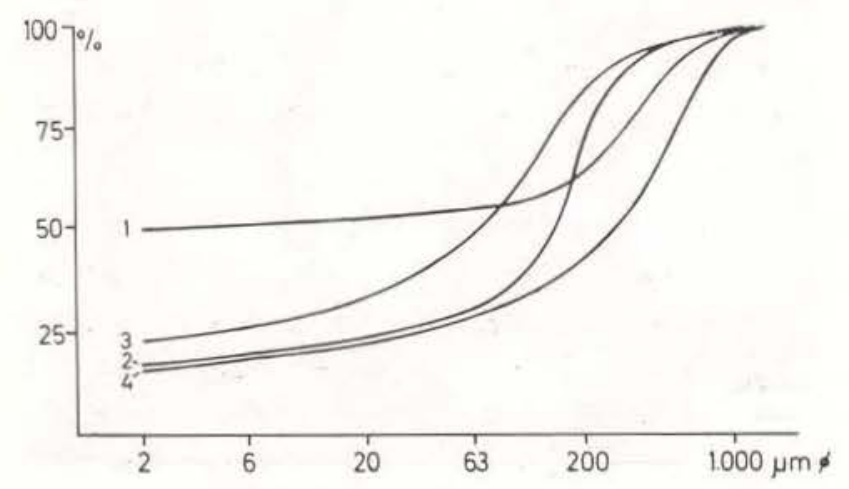

Fig. 3 - Distribuição granulométrica em quatro unidades selecionadas de uma sondegem de $10 \mathrm{~m}$ de profundidade, da área estudada

\begin{tabular}{ccccc} 
Unidade & $\begin{array}{c}\text { Profundidade } \\
\text { sob o solo }(\mathrm{m})\end{array}$ & Argila & Silt & $\begin{array}{c}\text { Areia } \\
(\%)\end{array}$ \\
1 & $0,3-0,6$ & 51 & 4 & 43 \\
2 & $3,0-3,5$ & 21 & 12 & 67 \\
3 & $5,6-5.8$ & 29 & 26 & 45 \\
4 & $9,0-10,0$ & 18 & 16 & 66 \\
\hline
\end{tabular}

cerca de $20 \%$ de fração de argila $(<2 \mu \mathrm{m})$, enquanto a amostra mais superficial contém cerca de $50 \%$ de fração de argila. Todas essas amostras apresentam uma distribuição bimodal, mostrando uma máxima na fração de argila e outra na fração $>125 \mu \mathrm{m}$. A fração de argila é constituída por mais de $90 \%$ de caulinita; as amostras mais profundas contêm também quartzo e ilita, ao passo que a amostra de menor profundidade contém alguma gipsita, quantidades menores de lâminas de silicato semelhantes à sudoita, e traços de quartzo.

A caolinita das 3 amostras de maior profundidade está mais bem ordenada ao longo do eixo $B$, enquanto que a da amostra mais alta se encontra bastante mal ordenada ao longo do mesmo eixo.

As fraçōes $>2 \mu \mathrm{m}$ são constituídas sobreduto por quartzo.

\section{DISCUSSÃo}

A distribuição granulométrica bimodal das amostras estudadas e sua composição mineralógica indicam que esse material era cons- 
tituído por uma proporção quase igual de quartzo e feldspato na mesma variação de tamanho (tamanho médio $=$ aproximadamente $0,2 \mathrm{~mm}$ ). O feldspato, durante a desagregação, modifica-se em caulinita, com uma granulometria de cerca de $2 \mu \mathrm{m}$. O clima tropical úmido, e, por conseguinte, com altas taxas de precipitação, existente com certeza nessa área desde o Plioceno, causou profunda desagregação das rochas-matrizes nas áreas drenadas do rio Negro e Tarumã-Mirim. Isto provocou a modificação do feldspato em caulinita, pelo menos, acima das profundidades correspondentes ao nível do igapó no TarumãMirim. O sedimento originalmente constituído por feldspato (cerca de $30 \%$ ), do qual resulta o sedimento do igapó investigado, não pode ter sido depositado durante o Pleistoceno ou Holoceno, porque as áreas drenadas do rio em questão não contêm nenhum feldspato.

A caulinita bem ordenada da parte mais baixa do perfil, modificou-se próximo à superfície, sob influência de fortes intemperismos, em caulinita menos bem orientada. Formouse então gipsita e o já citado mineral, semeIhante à sudoita (Irion, 1976a).

A sucessão mineralógica deste perfil é semelhante ao perfil erodido da vizinha formação Barreira (Cretáceo-Terciário) e pode ser designada como pertencente a essa formação (Irion, 1978). A sucessão de minerais do solo indica que a área de floresta é muito antiga. Superfícies geomorfológicas semelhantes, por ex. na parte mais baixa do rio Purus, no lago Aiapuá, muito provavelmente desenvolveramse, durante o último período interglacial, anterior a 100.000 anos. Essas superfícies apresentam um grau muito mais baixo de erosão (Irion, 1976b).

Esse fato sugere que a superfície de floresta do Tarumã-Mirim se desenvolveu durante o início do Pleistoceno ou mesmo no Plioceno.

Durante o Pleistoceno, o nível da água dos rios da Amazônia Central era atingido pelas mudanças do nível do mar (Klammer, 1976; Irion, 1976c). Durante as fases glaciais, os rios recortaram seus vales profundamente no sedimento mole (Sioli, 1968). Durante os períodos em que o nível do mar estava alto, esses vales, nas regiões de rios de água branca (rios com matéria em suspensão), eram preenchidos pelo sedimento; desse modo, nas regiões de rios de água clara e preta (pobre em matéria em suspensão), os vales eram apenas parcialmente preenchidos, e isso conduziu ao desenvolvimento de "lagos de ria" (Sioli, 1968), nas áreas do curso inferior dos rios. O rio Tarumã-Mirim pertence a esse último grupo.

\section{Conclusões}

A área examinada estava originalmente coberta por uma floresta de terra firme, exposta - há cerca de um milhão de anos antes do presente às flutuações anuais do nível da água do rio Negro, durante períodos de elevação do nível do mar. O último grande período de inundação sofrido pela área estudada provavelmente ocorreu por volta de 100.000 anos atrás, durante um tempo de aproximadamente 20.000-30.000 anos, outros, provavelmente, ocorreram há cerca de $60.000 \mathrm{e}$ 35.000 anos, durante um período de aproximadamente 10.000 anos.

Durante o último milhão de anos, os períodos anuais de inundação foram causando gradualmente a formação da presente vegetação, a qual é típica para florestas inundáveis de áreas de água preta (= Igapó; cf. Adis \& Rodrigues, $s / d$ ). De acordo com Ellenberg (comunicação verbal), esta vegetação poderia ter sido reprimida pela vegetação de terra fir. me durante períodos em que a água do mar estava baixa (face à pressão de competição).

Há 6.000 anos, o nivel do mar atingiu uma altura de cerca de $5 \mathrm{~m}$ abaixo de NN (Woldstedt, 1969). Como resultado aumentou o nivel da água do sistema de rios; desde então, a floresta estudada é inundada anualmente entre março/abril e agosto/setembro. Como o nível do mar sobe muito lentamente, a duração anual da inundação era possivelmente menor no início. 
As primeiras florestas de inundação de senvolveram-se, aproximadamente, há $1 \mathrm{mi}$ Ihão de anos, nas adjacências das margens do rio Negro, conseqüentemente também na con fluência dos rios Tarumã-Mirim e rio Negro. Essas florestas têm estado, desde então, ex. postas às flutuações anuais do nível da água do rio Negro. Os períodos de inundação conduziram a adaptações dos artrópodos do solo

\section{SUMMARY}

An attempt has been made to understand the ground-living arthropods behaviour in relation to the sedimentological evolution of rio Tarumă-Mirim, near Manaus, Amazonas, during the quaternary period. The area examined originally was covered by a Terra-Firme forest, exposed since about I million years B.P. to the annual water-level fluctuations of the rio Negro during periods of high sea-levei. The last annual inundations of the area studied probably took place 100.000 years ago for a period of approximately $20.000-30.000$ years; others probably occurred about 60.000 and 35.000 years ago for a period of approximately 10.000 years. The annual inundation-periods within the last 1.000.000 years gradually caused the formation of the present vegetation which is typical for inundation-forests in blackwater areas. According to Ellenberg (verbal communication), this vegetation may have been displayed periodically by terra-firme vegetation during low seawater periods (becauss of competition pressure). 6000 years ago the sea-level reached a height of abcut $5 \mathrm{~m}$ below NN. As a result of back pressure at this sea-level position, the water-level of the river-system increased; since then the examined forest in annually inundated between March/April and August/September. Because the sea-level rose very slowly, the annual duration of inundation was possibly shorter in the beginning. The first inundation-forests arose approximately I million years ago in the direct surroundings of the rio Negro bank, consequently at the confluence of rio Tarumã Mirim and rio Negro too. These forests have been exposed since that time to the annual fluctuations of the rio Negro water-level. The inundation-periods led to adaptions of ground-living arthropods.

\section{BIELIOGRAFIA}

ADIs, J.

1977 - Programa mínimo para análise de ecossis. temâs: Artrópodos terrestres em florestas inundáveis da Amazônia Central. Acta Amazonica, 7(2) : 223-229.
AdIS, J. \& RODRIGUES, W.A.

s/d - Programa mínimo para análise de ecossistemas: Inventário florestal da Area Experimental do rio Tarumã-Mirim. Acta Ama. zonica, (em preparo).

BECK, L.

1960 - Zum jahreszeitlichen Massenwechsel zweier Oribatidenarten (Acari) im neotropischen Uberschwemmungs-wald, Verh. dtsch. Zool. Ges. Innsbruck: 535-540.

1971 - Bodenzoologische Gliederung und Charakterisierung des amazonischen Regenwaldes. Amazoniana, 3(1) : 69-132.

1972 - Der Einfluss der jahresperiodischen Oberflutungen auf den Massenwechsel der Bodenarthropoden im zentral-amazonischen Regenwaldgebiet. Pedobiologia, 12:133-148.

1976 - Zum Massenwechsel der Makro-Arthropodenfauna des Bodens in Oberschwemmungswäldern des zentralen Amazonasgebietes. Amazoniana, $6(1): 1-20$.

IrION, G .

1976a - Mineralogisch-geochemische Untersuchungen an der pelitischen Fraktion amazonischer Oberböden und Sedimente. Biogeographica, $7: 7-25$.

$1976 \mathrm{~b}$ - Quaternary Sediments of the upper Amazon lowlands of Brazil. Biogeographica, 7 : 163-167.

1976c-Die Entwicklung des zentral-und oberamazonischen Tieflands im Spät-Pleistozän und im Holozän. Amazoniana, $6(1)$ : 67-79.

1978 - Soil Infertility in the Amazonian Rain Forest. Die Naturwissenschaften, 65 : 515-519.

IRMLER, U.

1975 - Ecological Studies of the Aquatic Soil Invertebrates in Three Inundation Forests of Central Amazonia. Amazoniana, 5(3) : 337-409.

1976 - Zusammensetzung, Besiedelungsdichte und Biomasse der Makrofauna des Bodens in der emersen und submersen Phase zentralamazonischer überschwemmungswälder. Biogeographica, $7: 79-99$.

1978 - Die Struktur der Carabiden-und Staphyliniden-gesellschaften in zentralamazonischen Oberschwemmungswäldern. Amazoniana, $6(3): 301-326$.

$\mathrm{s} / \mathrm{d}$ - Abundance fluctuations and habltat changes of soil beetles in Central Amazonian inundation forests (Coleoptera: Carabidae, Staphylinidae). Stud. neotrop. Fauna \& Environ. (in press). 
KLAMMER, G.

1976 - Zur jungquartären Reliefgeschichte des Amazonastales. Z. Geomorph. N.F., 20(2) : 149-170.

MONTGOMERY, G.; LUBIN, Y. \& ADIS, J.

$\mathrm{s} / \mathrm{d}$ - Diversity and Biomass of Arthropods in the Canopy of Inundated and Terra Firme Forests near Manaus, Brasil: A preliminary Report (in prep.).

SCHALLER, F

1969 - Zur Frage des Formensehens bei Collembolen. Verh. Dtsch. Zool. Ges. Innsbruck, 368-375.
1973 - Zur Ernährungsbiologie neotropischer Bodentiere. Die Naturwissenschaften, 4: 203.

Sioli, H.

1968 - Zur Ökologie des Amazonas-Gebiets. Biogeography and Ecology in South America I. Monogr. Biol., 18:137-170.

WOLSTEDT, $\mathbf{P}$ 1969 - Quartär. - Stuttgart.

(Aceito para publicação em 29/02/79) 